\title{
Some New Trigonometric $\alpha$-Order Fuzzy Entropies
}

Farzad Farhadyar

Department of Statistics

Faculty of Mathematical Sciences and Computer,

Shahid Chamran University of Ahvaz, Ahvaz-Iran

Email: Farzad.Farhadyar@gmail.com

Mohammad Reza Zadkarami

Department of Statistics

Faculty of Mathematical Sciences and Computer,

Shahid Chamran University of Ahvaz, Ahvaz-Iran

Email: zadkarami_m@scu.ac.ir or zadkarami@yahoo.co.uk

Rahim Chinipardaz

Department of Statistics

Faculty of Mathematical Sciences and Computer,

Shahid Chamran University of Ahvaz, Ahvaz-Iran

Email: chinipardaz_r@scu.ac.ir

\begin{abstract}
In this paper, new $\alpha$-order trigonometric and inverse trigonometric fuzzy entropies are proposed and the fuzzy entropy axiomatic requirements are satisfied for the new fuzzy entropies. A comparison of the new fuzzy entropies is done with several widely used fuzzy entropies in order to find the most fuzziness entropy. The results indicate that the new proposed $\alpha$-order fuzzy entropy provides larger entropy value than those other fuzzy entropies which were defined in the paper.
\end{abstract}

Keywords: Fuzzy entropy; Entropy axiomatic; Trigonometric; $\alpha$-order fuzzy entropy

\section{Introduction}

The fuzzy sets firstly defined by Zadeh (1965) to use for modeling non-statistical and vague information data (Chatterjee et al., 2017; Campion et al., 2018). Recently, the fuzzy sets become an interesting research topics in the applied sciences, e.g. engineering, image processing, medical sciences and so forth which are involving in using vague information (Fan and May, 2002; Hooda and Mishra, 2015; Dos et al., 2016).

The fuzzy entropy plays an important role in the fuzzy set theory because it is a fuzziness measure of the fuzzy sets. Some authors used the fuzzy entropy in statistical inference, e.g. estimation and testing hypothesis concepts (Zamanzade and Arghami, 2011; Zamanzade and Arghami, 2012; Zamanzade, 2014; Zamanzade and Mahdizadeh, 2016; Zamanzade and Mahdizadeh, 2017). Zadeh (1968) proposed a fuzzy entropy based on the probabilistic framework. However, the fuzzy entropy based on the concept of the Shannon statistical entropy (Shannon, 1948) is defined by De Luca and Termini (1972). They provided an axiomatic framework to define new fuzzy entropy measures (FEM). Several studies employed these axioms to propose new fuzzy entropy.

Bhandari and Pal (1993) provided a $\alpha$-order type of FEM. There exists some new fuzzy $\alpha-$ order entropies proposed by Kapur (1997), Fan and Ma (2002) and Al-Talib and Al-Nasser (2018). 
Fuzzy entropies based on the trigonometric functions are also considered by several authors e.g., Parkash et al. (2008) and Verma (2015). Hooda and Mishra (2015) defined several trigonometric fuzzy entropies based on sinus and cosine functions and along with studying their properties.

In this paper, we generalized some $\alpha$-order trigonometric fuzzy entropies and studied their properties. We found out the measure of the new $\alpha$-order inverse trigonometric fuzzy entropy, based on arcsin function, is larger than that of the $\alpha$-order fuzzy entropies and $\alpha$ order trigonometric entropies for different values of $\alpha$.

This paper is organized as follows. In Section 2, some concepts and axioms of the fuzzy entropy and some fuzzy entropy measures, which are proposed by authors, are presented. We proposed several new $\alpha$-order trigonometric and inverse trigonometric entropies in Section 3. The measurement comparison of the new fuzzy entropies with some widely used fuzzy entropies are done in Section 4 and Section 5 includes some conclusions.

\section{Fuzzy Entropy Axioms}

In this section, some concepts and axioms of the fuzzy entropy are presented. Let $\mu_{A}$ denote the fuzzy membership function of the finite set $A=\left\{x_{i} ; i=1,2, \ldots, n\right\}$ where $\mu_{A\left(x_{i}\right)}$ is the value of the membership function of the element $x_{i}$ form $A$ and, the fuzzy entropy of $\mu_{A}$ is denoted by $H\left(\mu_{A}\right)$. Using the Shannon probabilistic entropy (1948), De Luca and Termini (1972) defined the fuzzy entropy

$$
H\left(\mu_{A}\right)=-K \sum_{i=1}^{n}\left[\mu_{A\left(x_{i}\right)} \log \mu_{A\left(x_{i}\right)}+\left(1-\mu_{A\left(x_{i}\right)}\right) \log \left(1-\mu_{A\left(x_{i}\right)}\right)\right]
$$

for some constant $K$ equal to $1 / n$. They also provided following axiomatic requirements that are satisfied to propose new fuzzy entropy measure, as well.

Axiom 1. Sharpness: $H\left(\mu_{A}\right)=0$ if and only if $A\left(x_{i}\right)$ is a crisp set for each $i$. $A$ is crisp if $\mu_{A\left(x_{i}\right)}=0$ or 1 for each $i=1,2, \ldots, n$.

Axiom 2. Maximality: $H\left(\mu_{A}\right)$ has a unique maximum value at $\mu_{A\left(x_{i}\right)}=0.5$.

Axiom 3. Resoltions: $H\left(\mu_{A}\right) \leq H\left(\mu_{A}^{*}\right)$ where $\mu_{A\left(x_{i}\right)}$ is crisper than $\mu_{A\left(x_{i}\right)}^{*}$.

A fuzzy set $A^{*}$ is called a sharpened version of the fuzzy set $A$ (or crisper than $A$ ) if for $\forall i, \mu_{A\left(x_{i}\right)}{ }^{*} \leq \mu_{A\left(x_{i}\right)}$ for $0 \leq \mu_{A\left(x_{i}\right)} \leq 0.5$ and $\mu_{A\left(x_{i}\right)} \leq \mu_{A\left(x_{i}\right)}{ }^{*}$ for $0 \leq \mu_{A\left(x_{i}\right)} \leq 0.5$.

Axiom 4. Symmetry: $H\left(\mu_{A}\right)=H\left(1-\mu_{A}\right)$ for each $\mu_{A\left(x_{i}\right)}$.

Several generalized fuzzy entropies were defined by authors e.g., Bhandari and Pal (1993) proposed a $\alpha$-order fuzzy entropy based on the Rényi's entropy (1961) as follows:

$$
H_{\alpha}^{B P}\left(\mu_{A}\right)=(1-\alpha)^{-1} \sum_{i=1}^{n} \log \left[\mu_{A\left(x_{i}\right)}^{\alpha}+\left(1-\mu_{A\left(x_{i}\right)}\right)^{\alpha}\right) ; \alpha \neq 1, \alpha>0 .
$$

Furthermore, Kapur [11] proposed following $\alpha$-order FEM

$H_{\alpha}^{K}\left(\mu_{A}\right)=(1-\alpha)^{-1} \sum_{i=1}^{n}\left[\left\{\mu_{A\left(x_{i}\right)}^{\alpha}+\left(1-\mu_{A\left(x_{i}\right)}\right)^{\alpha}\right\}-1\right] ; \alpha \neq 1, \alpha>0$.

Some fuzzy entropy measures are suggested by authors, e.g., Al-Talib and Al-Nasser (2018) defined a $\alpha$-order entropy measure

$$
H_{\alpha}^{N T}\left(\mu_{A}\right)=\sum_{i=1}^{n}\left(\frac{\mu_{A\left(x_{i}\right)^{\frac{\alpha}{2}}}\left(1-\mu_{\left.A\left(x_{i}\right)\right)^{\frac{\alpha}{2}}}\right.}{\mu_{A\left(x_{i}\right)} e^{-\alpha *\left(1-\mu_{A\left(x_{i}\right)}\right)}+\left(1-\mu_{A\left(x_{i}\right)}\right) e^{-\alpha * \mu_{A\left(x_{i}\right)}}}\right)^{\frac{1}{\alpha}} ; \alpha>0 .
$$


Some trigonometric fuzzy entropies are also proposed by Hood and Mishra (2015) as follows

$$
\begin{gathered}
H^{S}\left(\mu_{A}\right)=\sum_{i=1}^{n}\left[\operatorname{Sin}\left(\frac{\pi \mu_{A\left(x_{i}\right)}}{2}\right)+\operatorname{Sin}\left(\frac{\pi\left(1-\mu_{A\left(x_{i}\right)}\right)}{2}\right)-1\right] \\
H^{C}\left(\mu_{A}\right)=\sum_{i=1}^{n}\left[\operatorname{Cos}\left(\frac{\pi \mu_{A\left(x_{i}\right)}}{2}\right)+\operatorname{Cos}\left(\frac{\pi\left(1-\mu_{A\left(x_{i}\right)}\right)}{2}\right)-1\right] \\
H^{C S}\left(\mu_{A}\right)=\sum_{i=1}^{n} \operatorname{Cos}\left(\frac{\left|2 \mu_{A\left(x_{i}\right)}-1\right| \pi}{2}\right)=\sum_{i=1}^{n} \frac{\operatorname{Sin}\left(\frac{2 \pi \mu_{A\left(x_{i}\right)}}{2}\right)+\operatorname{Cos}\left(\frac{\pi\left(1-2 \mu_{A\left(x_{i}\right)}\right)}{2}\right)}{2}
\end{gathered}
$$

A $\alpha$-order inverse trigonometric fuzzy entropy is also defined

$$
H_{\alpha}^{\operatorname{Arctan}}\left(\mu_{A}\right)=\frac{2}{(1-\alpha)} \sum_{i=1}^{n}\left[\operatorname{Arctan}\left[\mu_{A\left(x_{i}\right)}{ }^{\alpha}+\left(1-\mu_{A\left(x_{i}\right)}\right)^{\alpha}-\frac{\pi}{4}\right]\right.
$$

Some authors proposed fuzzy entropy measures to be applicable in special cases, e.g., $\mathrm{Hu}$ and $\mathrm{Yu}$ (2004) and Gupta and Sheoran (2014).

\section{New $\alpha$-order Trigonometric FEM}

From mathematical point of views, trigonometric measures have important properties in the modeling of geometry applications. In this section, some new $\alpha$-order fuzzy entropy measures based on trigonometric functions are proposed as follows.

$$
\begin{aligned}
& H_{\alpha}^{S}\left(\mu_{A}\right)=\sum_{i} \frac{\operatorname{Sin}^{\alpha}\left[\frac{\pi \mu}{2} A\left(x_{i}\right)\right]+\operatorname{Sin}^{\alpha}\left[\frac{\pi\left(1-\mu \mu\left(x_{i}\right)\right.}{2}\right]-1}{-1+2^{1-\frac{\alpha}{2}}} ; \alpha>0, \alpha \neq 2 \text {, } \\
& H_{\alpha}^{C}\left(\mu_{A}\right)=\sum_{i} \frac{\operatorname{Cos}^{\alpha}\left[\frac{\pi \mu}{2}\right]+\operatorname{Cos}^{\alpha}\left[\frac{\pi(1-\mu}{2} \frac{\left.x_{i}\right)^{)}}{2}\right]-1}{-1+2^{1-\frac{\alpha}{2}}} ; \alpha>0, \alpha \neq 2 \\
& H_{\alpha}^{S C}\left(\mu_{A}\right)=\sum_{i} \frac{\operatorname{Sin}^{\alpha}\left[\frac{2 \pi \mu}{2} A\left(x_{i}\right)\right]+\operatorname{Cos}^{\alpha}\left[\frac{\pi(1-2 \mu}{2} A\left(x_{i}\right)\right)}{2} ; \alpha>0 \text {, and } \\
& H_{\alpha}^{A r c s i n}\left(\mu_{A}\right)=\sum_{i} \frac{-\operatorname{Arcsin}\left[\mu_{A\left(x_{i}\right)}{ }^{\alpha}+\left(1-\mu_{A\left(x_{i}\right)}\right)^{\alpha}\right]+\frac{\pi}{2}}{\frac{\pi}{2}-\operatorname{Arcsin}\left[2^{1-\alpha}\right]} ; \alpha>1
\end{aligned}
$$

where $0 \leq \mu_{A\left(x_{i}\right)} \leq 1,(i=1,2, \ldots, n)$ denotes the fuzzy membership function.

Ultimately, we show that the new proposed fuzzy entropy measures satisfy all the entropy properties which are indicated in the preceding section.

Theorem 1. The FEM(1) satisfies four fuzzy entropy axiomatic requirements.

Proof: We show that required axioms 1-4 for fuzzy entropy measures are satisfied for $\operatorname{FEM}(1)$ after it is indicated that the FEM(1) is non-negative.

We have $2^{\frac{\alpha}{2}}>2$ for $\forall \alpha>2$ and thus $2^{1-\frac{\alpha}{2}}<1$. Therefore, the denominator of $H_{\alpha}^{S}\left(\mu_{A}\right)$ is negative. Moreover,

$$
\operatorname{Sup}_{0 \leq \mu_{A\left(x_{i}\right)} \leq 1}\left(\operatorname{Sin}^{\alpha}\left[\frac{\pi \mu_{A\left(x_{i}\right)}}{2}\right]+\operatorname{Sin}^{\alpha}\left[\frac{\pi\left(1-\mu_{A\left(x_{i}\right)}\right)}{2}\right]\right)=\left[(0)^{\alpha}+(1-0)^{\alpha}\right]=1 \text { for } \forall \alpha>2 \text {. }
$$

Therefore, for $\forall \alpha>2$ we have $\operatorname{Sin}^{\alpha}\left[\frac{\pi \mu_{A\left(x_{i}\right)}}{2}\right]+\operatorname{Sin}^{\alpha}\left[\frac{\pi\left(1-\mu_{A\left(x_{i}\right)}\right)}{2}\right]-1 \leq 0$ and hence, the nominator of $H_{\alpha}^{S}\left(\mu_{A}\right)$ is non-negative. Then $H_{\alpha}^{S}\left(\mu_{A}\right) \geq 0$. 
However, $2^{\frac{\alpha}{2}}<2$ for $\forall \alpha<2$ and then $2^{1-\frac{\alpha}{2}}<1$ and the $H_{\alpha}^{S}\left(\mu_{A}\right)$ denominator is positive. Furthermore, $\operatorname{Inf}_{0 \leq \mu_{A\left(x_{i}\right)} \leq 1}\left(\operatorname{Sin}^{\alpha}\left[\frac{\pi \mu_{A\left(x_{i}\right)}}{2}\right]+\operatorname{Sin}^{\alpha}\left[\frac{\pi\left(1-\mu_{A\left(x_{i}\right)}\right)}{2}\right]\right)=\left[(0)^{\alpha}+(1-0)^{\alpha}\right]=1$ for $\forall \alpha<2$ and therefore, $\operatorname{Sin}^{\alpha}\left[\frac{\pi \mu_{A\left(x_{i}\right)}}{2}\right]+\operatorname{Sin}^{\alpha}\left[\frac{\pi\left(1-\mu_{A\left(x_{i}\right)}\right)}{2}\right]-1 \geq 0$. Then, the nominator of $H_{\alpha}^{S}\left(\mu_{A}\right)$ is non-negative and thus, $H_{\alpha}^{\operatorname{Arcsin}}\left(\mu_{A}\right) \geq 0$.

Sharpness: we have $H_{\alpha}^{S}\left(\mu_{A}\right)=0$ by replacing the value of $\mu_{A\left(x_{i}\right)}=0$ or 1 in (1). This result is a straight forward for every $\alpha \geq 1$. Conversely, suppose $H_{\alpha}^{S}\left(\mu_{A}\right)=0$, then

$$
\operatorname{Sin}^{\alpha}\left[\frac{\pi \mu_{A\left(x_{i}\right)}}{2}\right]+\operatorname{Sin}^{\alpha}\left[\frac{\pi\left(1-\mu_{A\left(x_{i}\right)}\right)}{2}\right]-1=0
$$

and, $\mu_{A\left(x_{i}\right)}$ should be zero or one.

Maximality: If differentiate $\operatorname{FEM}(1)$ with respect to $\mu_{A\left(x_{i}\right)}$, then we have $\frac{\partial H_{\alpha}^{S}\left(\mu_{A}\right)}{\partial \mu_{A\left(x_{i}\right)}}$

$=\frac{-\frac{1}{2} \pi \alpha \operatorname{Cos}\left[\frac{1}{2} \pi\left(1-\mu_{A\left(x_{i}\right)}\right)\right] \operatorname{Sin}\left[\frac{1}{2} \pi\left(1-\mu_{A\left(x_{i}\right)}\right)\right]^{-1+\alpha}+\frac{1}{2} \pi \alpha \operatorname{Cos}\left[\frac{\pi \mu_{A\left(x_{i}\right)}}{2}\right] \operatorname{Sin}\left[\frac{\pi \mu_{A\left(x_{i}\right)}}{2}\right]^{-1+\alpha}}{-1+2^{1-\frac{\alpha}{2}}}$

Thus, by solving the equation $\frac{\partial H_{\alpha}^{S}\left(\mu_{A}\right)}{\partial \mu_{A\left(x_{i}\right)}}=0$, it is obtained $\mu_{A\left(x_{i}\right)}=0.5$ and if assume $0<\mu_{A\left(x_{i}\right)}<0.5$, then we get

$\frac{\partial H_{\alpha}^{S}\left(\mu_{A}\right)}{\partial \mu_{A\left(x_{i}\right)}}>0, \forall \alpha>0, \alpha \neq 2$ and $\frac{\partial H_{\alpha}^{S}\left(\mu_{A}\right)}{\partial \mu_{A\left(x_{i}\right)}}<0, \forall \alpha>0, \alpha \neq 2$

where, $0.5<\mu_{A\left(x_{i}\right)}<1$. Furthermore, for $\mu_{A\left(x_{i}\right)}=0.5$, we get

$$
\frac{\partial H_{\alpha}^{S}\left(\mu_{A}\right)}{\partial \mu_{A\left(x_{i}\right)}}=0, \forall \alpha>0, \alpha \neq 2
$$

Therefore, $H_{\alpha}^{S}\left(\mu_{A}\right)$ is a concave function of $\mu_{A\left(x_{i}\right)}$ which has a global maximum at the point $\mu_{A\left(x_{i}\right)}=0.5$. Thus, $H_{\alpha}^{S}\left(\mu_{A}\right)$ attends its maximum if and only if $\mathrm{A}$ is the fuzziest set, i.e. $\mu_{A\left(x_{i}\right)}=0.5 \forall i=1, \ldots n$, which show the uniqueness of the maximum value.
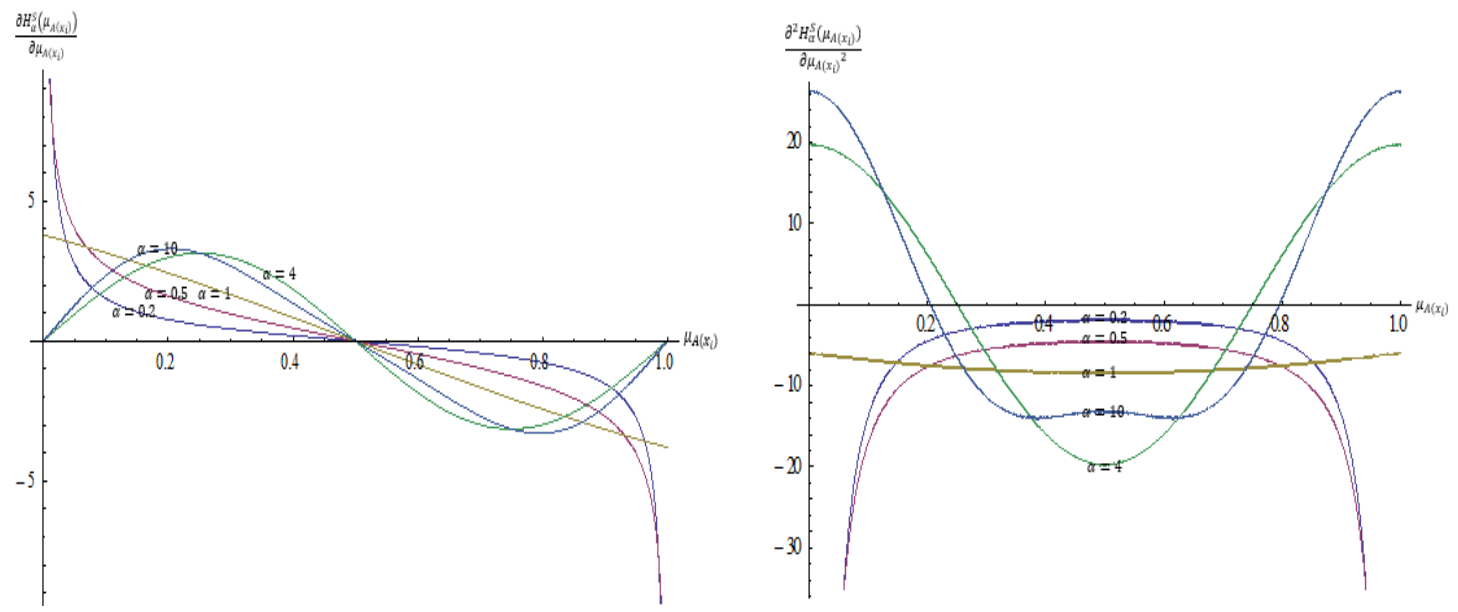
Figure 1: Plots of the first (left) and second (right) derivative of $H_{\alpha}^{S}\left(\mu_{A}\right)$ at different values of $\alpha$.

Now, consider the second derivative of (1) as follows.

$$
\begin{aligned}
& \frac{\partial^{2} H_{\alpha}^{S}\left(\mu_{A}\right)}{\partial \mu_{A\left(x_{i}\right)}{ }^{2}} \\
& =\frac{\frac{1}{4} \pi^{2}(-1+\alpha) \alpha \operatorname{Cos}\left[\frac{1}{2} \pi\left(1-\mu_{A\left(x_{i}\right)}\right)\right]^{2} \operatorname{Sin}\left[\frac{1}{2} \pi\left(1-\mu_{A\left(x_{i}\right)}\right)\right]^{-2+\alpha}-\frac{1}{4} \pi^{2} \alpha \operatorname{Sin}\left[\frac{1}{2} \pi\left(1-\mu_{A\left(x_{i}\right)}\right)\right]^{\alpha}}{-1+2^{1-\frac{\alpha}{2}}} \\
& +\frac{\frac{1}{4} \pi^{2}(-1+\alpha) \alpha \operatorname{Cos}\left[\frac{\pi \mu_{A\left(x_{i}\right)}}{2}\right]^{2} \operatorname{Sin}\left[\frac{\pi \mu_{A\left(x_{i}\right)}}{2}\right]^{-2+\alpha}-\frac{1}{4} \pi^{2} \alpha \operatorname{Sin}\left[\frac{\pi \mu_{A\left(x_{i}\right)}}{2}\right]^{\alpha}}{-1+2^{1-\frac{\alpha}{2}}}
\end{aligned}
$$

Therefore, we get

Where, $\left.\frac{\partial^{2} H_{\alpha}^{S}\left(\mu_{A}\right)}{\partial \mu_{A\left(x_{i}\right)^{2}}}\right|_{\mu_{A\left(x_{i}\right)}=0.5}<0, \forall \alpha>0, \alpha \neq 2$.

$$
\left.\frac{\partial^{2} H_{\alpha}^{S}\left(\mu_{A}\right)}{\partial \mu_{A\left(x_{i}\right)^{2}}}\right|_{\mu_{A\left(x_{i}\right)}=0.5}=\frac{-2^{-1-\frac{\alpha}{2}} \pi^{2} \alpha+2^{-2+\frac{2-\alpha}{2}} \pi^{2}(-1+\alpha) \alpha}{-1+2^{1-\frac{\alpha}{2}}} .
$$

Thus, the FEM(1) attends its maximum at $\mu_{A\left(x_{i}\right)}=0.5$. Figure 1 presents the graph of the first and second derivative of $H_{\alpha}^{S}\left(\mu_{A}\right)$ for different values of $\alpha$. As it is noticed from Figure 1 , the second derivative of $H_{\alpha}^{S}\left(\mu_{A}\right)$ is negative at $\mu_{A\left(x_{i}\right)}=0.5$. Therefore, the maximum value of $H_{\alpha}^{S}\left(\mu_{A}\right)$ happens at $\mu_{A\left(x_{i}\right)}=0.5$ which is equal to 1 .

The fuzzy entropy (1) for various values of the membership $\mu_{A\left(x_{i}\right)}$ for $\alpha=0.2,0.5,1,4$ and 10 is given in Table 1 . By taking a close look in Table 1 , the FEM $H_{\alpha}^{S}\left(\mu_{A}\right)$ is increasing function of $\mu_{A\left(x_{i}\right)}$ in the interval $[0,0.5]$ and decreasing function in the interval $[0.5,1]$ for diversity values of $\alpha$.

Figure 2 and Table 1 indicate that the fuzzy entropy measure $H_{\alpha}^{S}\left(\mu_{A}\right)$ is a decreasing function of $\alpha$ for values less than 4.09 and is an increasing function of $\alpha$ for values larger than 4.09 .

Table 1. The value of $\boldsymbol{H}_{\boldsymbol{\alpha}}^{S}\left(\boldsymbol{\mu}_{\boldsymbol{A}}\right)$ for $\boldsymbol{\alpha}=0.2,0.5,1,4$ and10.

\begin{tabular}{cccccc}
\hline$\mu_{A\left(x_{i}\right)}$ & $H_{\alpha=0.2}^{S}\left(\mu_{A}\right)$ & $H_{\alpha=0.5}^{S}\left(\mu_{A}\right)$ & $H_{\alpha=1}^{S}\left(\mu_{A}\right)$ & $H_{\alpha=4}^{S}\left(\mu_{A}\right)$ & $H_{\alpha=10}^{S}\left(\mu_{A}\right)$ \\
\hline 0.1 & 0.793881 & 0.571058 & 0.347943 & 0.0954915 & 0.124282 \\
0.2 & 0.901416 & 0.778997 & 0.627873 & 0.345492 & 0.420867 \\
0.3 & 0.959613 & 0.906022 & 0.832896 & 0.654508 & 0.729884 \\
0.4 & 0.990309 & 0.97702 & 0.957965 & 0.904508 & 0.933299 \\
0.5 & 1 & 1 & 1 & 1 & 1 \\
0.6 & 0.990309 & 0.97702 & 0.957965 & 0.904508 & 0.933299 \\
0.7 & 0.959613 & 0.906022 & 0.832896 & 0.654508 & 0.729884 \\
0.8 & 0.901416 & 0.778997 & 0.627873 & 0.345492 & 0.420867 \\
0.9 & 0.793881 & 0.571058 & 0.347943 & 0.0954915 & 0.124282 \\
1 & 0 & 0 & 0 & 0 & 0 \\
\hline
\end{tabular}



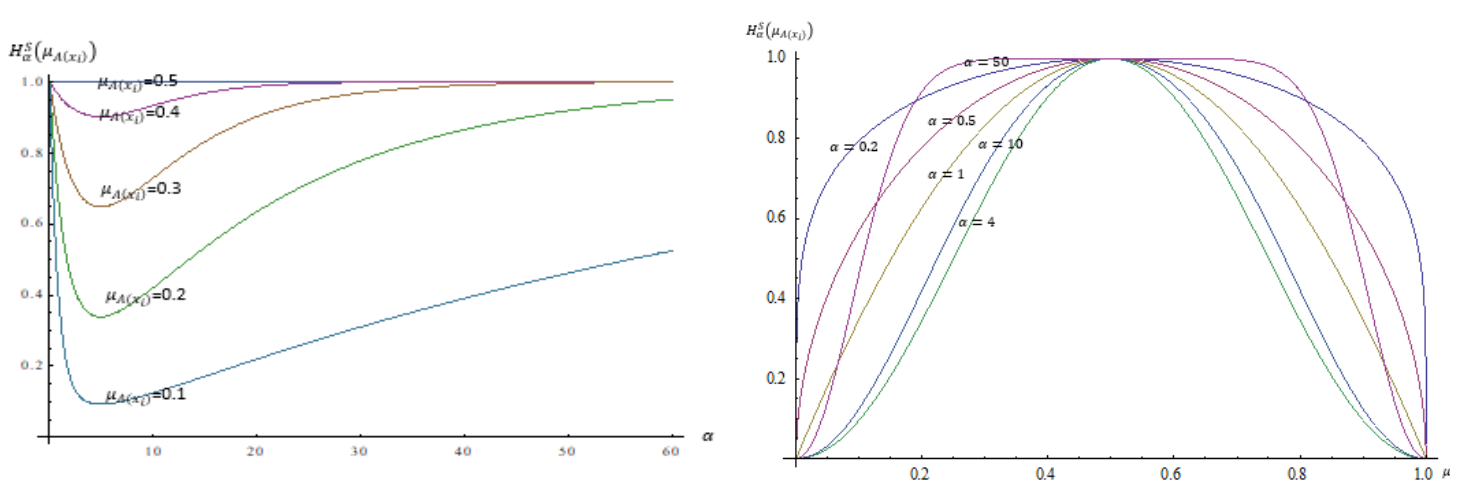

Figure 2: Plots of $H_{\alpha}^{S}\left(\mu_{A}\right)$ as a function of $\alpha$ for different values of $\mu_{A\left(x_{i}\right)}$ (left) and as a function of $\mu_{A\left(x_{i}\right)}$ for different values of $\alpha$ (right).

Resolutions: As it is presented in the maximality axiom, the fuzzy entropy $H_{\alpha}^{S}\left(\mu_{A}\right)$ has a unique maximum at $\mu_{A\left(x_{i}\right)}=0.5$ and thus, $H_{\alpha}^{S}\left(\mu_{A}\right)$ is a continuous concave function. Hence, $H_{\alpha}^{S}\left(\mu_{A}\right)$ monotonically increases for $\mu_{A\left(x_{i}\right)} \in[0,0.5]$ and monotonically decreases for $\mu_{A\left(x_{i}\right)} \in[0.5,1]$. Therefore, $H_{\alpha}^{S}\left(\mu_{A}^{*}\right) \leq H_{\alpha}^{S}\left(\mu_{A}\right)$ where $\mu_{A\left(x_{i}\right)}^{*}$ is a sharpened version of $\mu_{A\left(x_{i}\right)}$. Figure 2 illustrates the graph of $H_{\alpha}^{S}\left(\mu_{A}\right)$ for different values of $\alpha$.

Symmetry: We obtain $H_{\alpha}^{S}\left(1-\mu_{A}\right)=H_{\alpha}^{S}\left(\mu_{A}\right)$ when $1-\mu_{A\left(x_{i}\right)}$ instead of $\mu_{A\left(x_{i}\right)}$ in the $\operatorname{FEM}(1)$.

Therefore, four requirement axioms for the fuzzy entropy are satisfied for FEM(1) and the theorem 1 is proven.

Theorem 2. The FEM(2) satisfies all axiomatic requirements of the fuzzy entropy.

Proof: We verify to show that the axioms 1-4 are satisfied for $\operatorname{FEM}(2)$ and the $\operatorname{FEM}(2)$ is non-negative.

Since $0 \leq \mu_{A\left(x_{i}\right)} \leq 1$, then $0 \leq \pi \mu_{A\left(x_{i}\right)} \leq \pi$ and $-\frac{\pi}{2} \leq \frac{\pi\left(1-2 \mu_{A\left(x_{i}\right)}\right)}{2} \leq \frac{\pi}{2}$. Therefore, $\operatorname{Sin}\left[\frac{2 \pi \mu_{A\left(x_{i}\right)}}{2}\right] \geq 0$ and $\operatorname{Cos}\left[\frac{\pi\left(1-2 \mu_{A\left(x_{i}\right)}\right)}{2}\right] \geq 0$. Thus, $\frac{\left.\left.\operatorname{Sin}^{\alpha}\left[\frac{2 \pi \mu}{2}\right]+x_{i}\right)\right]+\operatorname{Cos}^{\alpha}\left[\frac{\pi\left(1-2 \mu_{A\left(x_{i}\right)^{)}}\right.}{2}\right]}{2} \geq 0$ for $\forall \alpha>1$ and then, $H_{\alpha}^{S C}\left(\mu_{A}\right) \geq 0$.

Sharpness: We can see by replacing the value $\mu_{A\left(x_{i}\right)}=0$ or 1 in the numerator of FEM (2), we get $H_{\alpha}^{C S}\left(\mu_{A}\right)=0$. This result is also satisfied for values $\alpha>1$. Conversely, assume $H_{\alpha}^{S}\left(\mu_{A}\right)=0$, then we obtain

$$
\operatorname{Sin}^{\alpha}\left[\frac{\pi 2 \mu_{A\left(x_{i}\right)}}{2}\right]+\operatorname{Cos}^{\alpha}\left[\frac{\pi\left(1-2 \mu_{A\left(x_{i}\right)}\right)}{2}\right]=0 .
$$

Therefore, $\mu_{A\left(x_{i}\right)}$ should be zero or one. 
Maximality: The first derivative of $\operatorname{FEM}(2)$ with respect to $\mu_{A\left(x_{i}\right)}$ is given by

$$
\begin{gathered}
\frac{\partial H_{\alpha}^{C S}\left(\mu_{A}\right)}{\partial \mu_{A\left(x_{i}\right)}}=\frac{1}{2}\left(\pi \alpha \operatorname{Cos}\left[\frac{1}{2} \pi\left(1-2 \mu_{A\left(x_{i}\right)}\right)\right]^{-1+\alpha} \operatorname{Sin}\left[\frac{1}{2} \pi\left(1-2 \mu_{A\left(x_{i}\right)}\right)\right]\right. \\
\left.\left.+\pi \alpha \operatorname{Cos}\left[\pi \mu_{A\left(x_{i}\right)}\right] \operatorname{Sin}\left[\pi \mu_{A\left(x_{i}\right)}\right]^{-1+\alpha}\right)\right)
\end{gathered}
$$

Then, we obtain $\mu_{A\left(x_{i}\right)}=0.5$ by solving the equation $\frac{\partial H_{\alpha}^{C S}\left(\mu_{A}\right)}{\partial \mu_{A\left(x_{i}\right)}}=0, \forall \alpha>0$.

Now, consider $0<\mu_{A\left(x_{i}\right)}<0.5$, then $\frac{\partial H_{\alpha}^{C S}\left(\mu_{A}\right)}{\partial \mu_{A\left(x_{i}\right)}}>0, \forall \alpha>0$. It can see if $\mu_{A\left(x_{i}\right)}$ belongs to the interval $(0.5,1)$, we have $\frac{\partial H_{\alpha}^{C S}\left(\mu_{A}\right)}{\partial \mu_{A\left(x_{i}\right)}}<0, \forall \alpha>0$ and, we get

$\frac{\partial H_{\alpha}^{C S}\left(\mu_{A}\right)}{\partial \mu_{A\left(x_{i}\right)}}=0, \forall \alpha>1$ for $\mu_{A\left(x_{i}\right)}=0.5$. Thus, $H_{\alpha}^{C S}\left(\mu_{A}\right)$ is a concave function which has a global maximum at $\mu_{A\left(x_{i}\right)}=0.5$. Therefore, the mesearse $H_{\alpha}^{C S}\left(\mu_{A}\right)$ attended its maximum value if and only if $\mathrm{A}$ is the fuzziest set, i.e. $\mu_{A\left(x_{i}\right)}=0.5 \forall i=1, \ldots n$, which results in the uniqueness of the maximum value.

Now consider the second derivative of $\operatorname{FEM}(2)$ as follows

$$
\begin{aligned}
\frac{\partial^{2} H_{\alpha}^{C S}\left(\mu_{A}\right)}{\partial \mu_{A\left(x_{i}\right)}{ }^{2}}=\frac{1}{2} & \left(-\pi^{2} \alpha \operatorname{Cos}\left[\frac{1}{2} \pi\left(1-2 \mu_{A\left(x_{i}\right)}\right)\right]^{\alpha}+\pi^{2}(-1\right. \\
& +\alpha) \alpha \operatorname{Cos}\left[\frac{1}{2} \pi\left(1-2 \mu_{A\left(x_{i}\right)}\right)\right]^{-2+\alpha} \operatorname{Sin}\left[\frac{1}{2} \pi\left(1-2 \mu_{A\left(x_{i}\right)}\right)\right]^{2}+\pi^{2}(-1 \\
& \left.+\alpha) \alpha \operatorname{Cos}\left[\pi \mu_{A\left(x_{i}\right)}\right]^{2} \operatorname{Sin}\left[\pi \mu_{A\left(x_{i}\right)}\right]^{-2+\alpha}-\pi^{2} \alpha \operatorname{Sin}\left[\pi \mu_{A\left(x_{i}\right)}\right]^{\alpha}\right)
\end{aligned}
$$

Thus, the equation: $\left.\frac{\partial^{2} H_{\alpha}^{C S}\left(\mu_{A}\right)}{\partial \mu_{A\left(x_{i}\right)}{ }^{2}}\right|_{\mu_{A\left(x_{i}\right)}=0.5}=-\pi^{2} \alpha$ is satisfied where,

$$
\left.\frac{\partial^{2} H_{\alpha}^{S}\left(\mu_{A}\right)}{\partial \mu_{A\left(x_{i}\right)^{2}}}\right|_{\mu_{A\left(x_{i}\right)}=0.5}<0, \forall \alpha>0 .
$$

Therefore, we see that the maximum of $\operatorname{FEM}(2)$ will attend at $\mu_{A\left(x_{i}\right)}=0.5$. For different values of $\alpha$, the graph of the first and second derivative of $H_{\alpha}^{C S}\left(\mu_{A}\right)$ are presented in Figure 3.
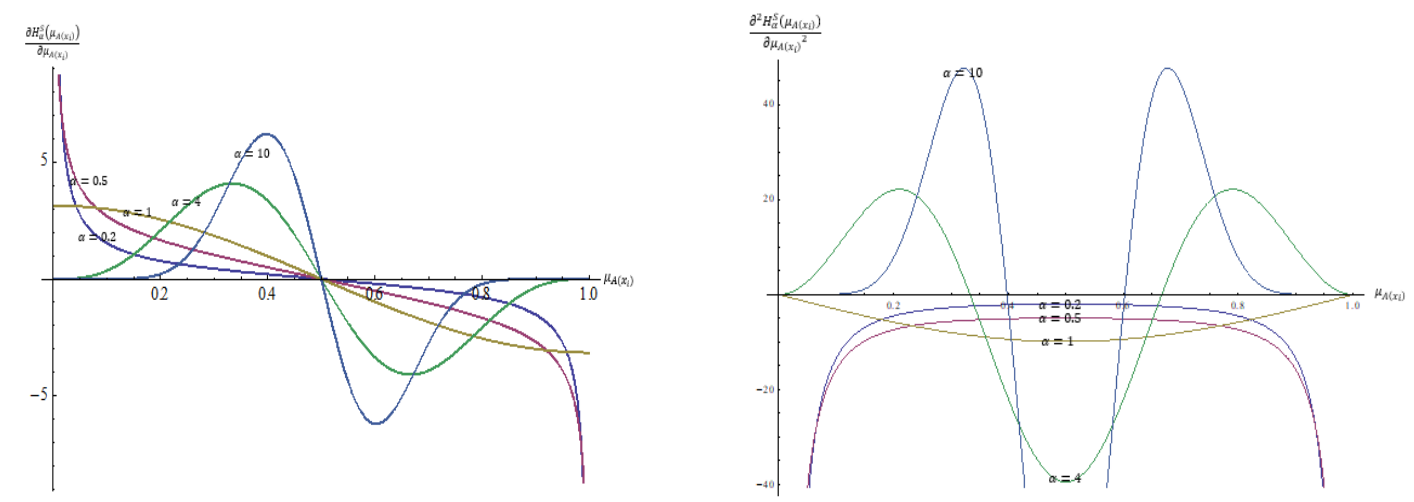

Pak.j.stat.oper.res. Vol.XV No. IV 2019 pp963-977 
Figure 3: Plots of the first (left) and second (right) derivative of $H_{\alpha}^{C S}\left(\mu_{A}\right)$ for $\alpha=0.2,0.5$,

$$
1,4 \text { and } 10 .
$$

As Figure 3 indicates, the second derivative of $H_{\alpha}^{C S}\left(\mu_{A}\right)$ for values $\alpha=0.2,0.5,1,4$ and 10 is negative. Therefore, the maximum value of $H_{\alpha}^{C S}\left(\mu_{A}\right)$ will be attended at $\mu_{A\left(x_{i}\right)}=0.5$ which is equal to 1 . Table 2 presents the numerical results of the measure (2) for different membership $\mu_{A\left(x_{i}\right)}$ values by considering $\alpha=0.2,0.5,1,4$ and 10 . We can see form Table 2 , the entropy measure (2) rises as a function of $\alpha$ for every membership function $\mu_{\mathrm{A}\left(\mathrm{x}_{\mathrm{i}}\right)}$. However, FEM H $\mathrm{H}_{\alpha}^{\mathrm{CS}}\left(\mu_{A}\right)$ increases for $0 \leq \mu_{\mathrm{A}\left(\mathrm{x}_{\mathrm{i}}\right)} \leq 0.5$ and declines for $0.5 \leq \mu_{\mathrm{A}\left(\mathrm{x}_{\mathrm{i}}\right)} \leq$ 1 for any given $\alpha=0.2,0.5,1,4$ and 10 .

Table 2. The values of $H_{\alpha}^{C S}\left(\mu_{A}\right)$ for $\alpha=0.2,0.5,1,4$ and 10.

\begin{tabular}{cccccc}
\hline$\mu_{A\left(x_{i}\right)}$ & $H_{\alpha=0.2}^{C S}\left(\mu_{\mu_{A}}\right)$ & $H_{\alpha=0.5}^{C S}\left(\mu_{A}\right)$ & $H_{\alpha=1}^{C S}\left(\mu_{A}\right)$ & $H_{\alpha=4}^{C S}\left(\mu_{A}\right)$ & $H_{\alpha=10}^{C S}\left(\mu_{A}\right)$ \\
\hline 0.1 & 0.790672 & 0.555893 & 0.309107 & 0.0091186 & $7.94006 \times 10^{-6}$ \\
0.2 & 0.899174 & 0.766672 & 0.587785 & 0.119364 & 0.00492251 \\
0.3 & 0.958499 & 0.899454 & 0.809017 & 0.428381 & 0.120109 \\
0.4 & 0.990014 & 0.975221 & 0.951057 & 0.818136 & 0.605429 \\
0.5 & 1 & 1 & 1 & 1 & 1 \\
0.6 & 0.990014 & 0.975221 & 0.951057 & 0.818136 & 0.605429 \\
0.7 & 0.958499 & 0.899454 & 0.809017 & 0.428381 & 0.120109 \\
0.8 & 0.899174 & 0.766672 & 0.587785 & 0.119364 & 0.00492251 \\
0.9 & 0.790672 & 0.555892 & 0.309017 & 0.0091186 & $7.94006 \times 10^{-6}$ \\
1 & 0 & 0 & 0 & 0 & 0 \\
\hline
\end{tabular}

Figure 4 shows that the measure $H_{\alpha}^{C S}\left(\mu_{A}\right)$ is a decreasing function of $\alpha$ which is greater than zero for different values of $\mu_{A\left(x_{i}\right)}$.
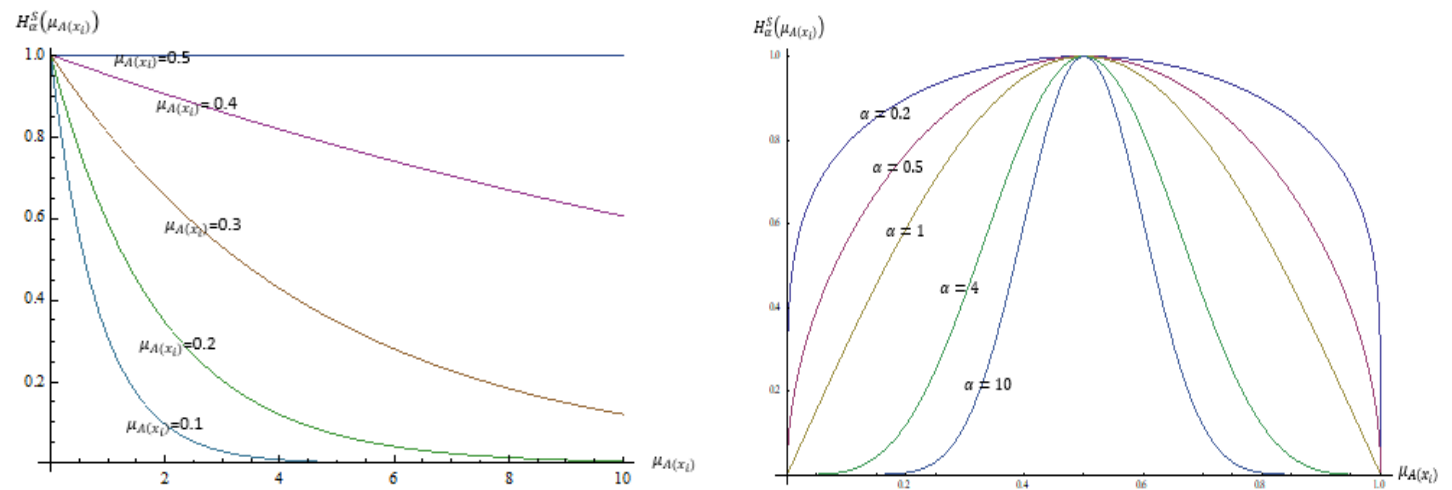

Figure 4: Plots of $H_{\alpha}^{C S}\left(\mu_{A}\right)$ as a function of $\alpha$ for different values of $\mu_{A\left(x_{i}\right)}$ (left) and as a function of $\mu_{A\left(x_{i}\right)}$ for $\alpha=0.2,0.5,1,4$ and 10 (right).

Resolutions: As discussed in the preceding axiom, the measure $H_{\alpha}^{C S}\left(\mu_{A}\right)$ has a unique maximum in $\mu_{A\left(x_{i}\right)}=0.5$ and furthermore, FEM $H_{\alpha}^{C S}\left(\mu_{A}\right)$ is a continuous concave function. Hence, FEM $H_{\alpha}^{C S}\left(\mu_{A}\right)$ is monotonically increasing for values: $0 \leq \mu_{A\left(x_{i}\right)} \leq 0.5$ and is monotonically decreasing for values: $0.5 \leq \mu_{A\left(x_{i}\right)} \leq 1$. Therefore, 
$H_{\alpha}^{C S}\left(\mu_{A}^{*}\right) \leq H_{\alpha}^{C S}\left(\mu_{A}\right)$ where $\mu_{A\left(x_{i}\right)}^{*}$ denotes the sharpened version of $\mu_{A\left(x_{i}\right)}$. Figure 4 presents the graph of $H_{\alpha}^{C S}\left(\mu_{A}\right)$ for different values of $\mu_{A\left(x_{i}\right)}$.

Symmetry: By substituting $1-\mu_{A\left(x_{i}\right)}$ instead of $\mu_{A\left(x_{i}\right)}$ in Equation (2), we get

$$
H_{\alpha}^{C S}\left(1-\mu_{A}\right)=H_{\alpha}^{C S}\left(\mu_{A}\right) \text {. }
$$

Therefore, four requirement axioms are satisfied for $\operatorname{FEM}(2)$ and theorem 3.2 is proven. Now, the four measurement requirement axioms are to be investigated for FEM(3).

Theorem 3. The FEM(3) satisfies all axiomatic requirements of the fuzzy entropy measures.

Proof: First, we show that the FEM(3) is nonnegative and secondly it will proved that the axioms 1-4 for fuzzy entropy measures are also satisfied for FEM(3).

We have $1>2^{\alpha-1}$ for $\forall \alpha>1$ because, $2^{\alpha}>2$ then, $\operatorname{Arcsin}[1]>\operatorname{Arcsin}\left[2^{\alpha-1}\right]$. Hence $\frac{\pi}{2}-\operatorname{Arcsin}\left[2^{1-\alpha}\right]>0$ and the $H_{\alpha}^{\operatorname{Arcsin}}\left(\mu_{A}\right)$ denominator is positive. Furthermore, $\operatorname{Sup}_{0<\mu_{A\left(x_{i}\right)}<1}\left[\mu_{A\left(x_{i}\right)}{ }^{\alpha}+\left(1-\mu_{A\left(x_{i}\right)}\right)^{\alpha}\right]=\left[\left(\frac{1}{2}\right)^{\alpha}+\left(1-\frac{1}{2}\right)^{\alpha}\right]<1$, for $\forall \alpha>1$. Therefore, $\operatorname{Arcsin}\left[\mu_{A\left(x_{i}\right)}^{\alpha}+\left(1-\mu_{A\left(x_{i}\right)}\right)^{\alpha}\right]<\operatorname{Arcsin}[1]=\frac{\pi}{2}$ and the nominator of $H_{\alpha}^{\operatorname{Arcsin}}\left(\mu_{A}\right)$ is non-negative. Thus, $\operatorname{FEM}(3)$ is non-negative.

Sharpness: We obtain $H_{\alpha}^{\operatorname{Arcsin}}\left(\mu_{A}\right)=0$ when it is replaced by the values: $\mu_{A\left(x_{i}\right)}=0$ or 1 in the numerator of $\operatorname{FEM}(3)$. This result is straight forward for each value of $\alpha>1$. Conversely, assume $H_{\alpha}^{S}\left(\mu_{A}\right)=0$, therefore,

$$
\operatorname{Arcsin}\left[\mu_{A\left(x_{i}\right)}^{\alpha}+\left(1-\mu_{A\left(x_{i}\right)}\right)^{\alpha}\right]+\frac{\pi}{2}=0
$$

thus, $\mu_{A\left(x_{i}\right)}=0$ or 1 .

Maximality: Getting differentiate form (3) with respect to $\mu_{A\left(x_{i}\right)}$ we obtain

$$
\frac{\partial H_{\alpha}^{A r c s i n}\left(\mu_{A}\right)}{\partial \mu_{A\left(x_{i}\right)}}=-\frac{\left.-\left(1-\mu_{A\left(x_{i}\right)}\right)^{-1+\alpha} \alpha+\mu_{A\left(x_{i}\right)}\right)^{-1+\alpha} \alpha}{\sqrt{1-\left(\left(1-\mu_{A\left(x_{i}\right)}\right)^{\alpha}+\mu_{\left.A\left(x_{i}\right)^{\alpha}\right)^{2}}\left(\frac{\pi}{2}-\operatorname{ArcSin}\left[2^{1-\alpha}\right]\right)\right.}} .
$$

By solving the equation $\frac{\partial H_{\alpha}^{\operatorname{Arcsin}}\left(\mu_{A}\right)}{\partial \mu_{A\left(x_{i}\right)}}=0, \forall \alpha>1$, we find that $\mu_{A\left(x_{i}\right)}=0.5$. Now, suppose $\mu_{A\left(x_{i}\right)} \in(0,0.5)$, then we have

We get

$$
\frac{\partial H_{\alpha}^{\operatorname{Arcsin}}\left(\mu_{A}\right)}{\partial \mu_{A\left(x_{i}\right)}}>0, \forall \alpha>1
$$

$$
\frac{\partial H_{\alpha}^{\operatorname{Arcsin}}\left(\mu_{A}\right)}{\partial \mu_{A\left(x_{i}\right)}}<0, \forall \alpha>1
$$

where, $\mu_{A\left(x_{i}\right)} \in(0.5,1)$, and furthermore, for $\mu_{A\left(x_{i}\right)}=0.5$, we have

$$
\frac{\partial H_{\alpha}^{\operatorname{Arcsin}}\left(\mu_{A}\right)}{\partial \mu_{A\left(x_{i}\right)}}=0, \forall \alpha>1
$$

Thus, $H_{\alpha}^{\operatorname{Arcsin}}\left(\mu_{A}\right)$ is a concave function of $\mu_{A\left(x_{i}\right)}$ with a global maximum at $\mu_{A\left(x_{i}\right)}=0.5$. Therefore, $H_{\alpha}^{\operatorname{Arcsin}}\left(\mu_{A}\right)$ get its maximum if and only if $\mathrm{A}$ is the fuzziest set, i.e. $\mu_{A\left(x_{i}\right)}=$ 
$0.5, \forall i=1, \ldots n$ and this proofs the uniqueness of the maximum value of $H_{\alpha}^{C S}\left(\mu_{A}\right)$. The graph of $\frac{\partial H_{\alpha}^{\operatorname{Arcsin}}\left(\mu_{A}\right)}{\partial \mu_{A\left(x_{i}\right)}}$ for different values of $\alpha$ is presented in Figure 5.

Furthermore, the second derivative of (3.3) is given by

$$
\begin{aligned}
\frac{\partial^{2} H_{\alpha}^{A r c s i n}\left(\mu_{A}\right)}{\left.\partial \mu_{A\left(x_{i}\right)}\right)^{2}}= & -\frac{\left(\left(1-\mu_{A\left(x_{i}\right)}\right)^{\alpha}+\mu_{A\left(x_{i}\right)}\right)\left(-\left(1-\mu_{A\left(x_{i}\right)}\right)^{-1+\alpha} \alpha+\mu_{A\left(x_{i}\right)}{ }^{-1+\alpha} \alpha\right)^{2}}{\left.\left(1-\left(\left(1-\mu_{A\left(x_{i}\right)}\right)^{\alpha}+\mu_{A\left(x_{i}\right)}\right)^{2}\right)^{2}\right)^{3 / 2}\left(\frac{\pi}{2}-\operatorname{ArcSin}\left[2^{1-\alpha}\right]\right)} \\
& -\frac{\left.\left(1-\mu_{A\left(x_{i}\right)}\right)^{-2+\alpha}(-1+\alpha) \alpha+\mu_{A\left(x_{i}\right)}\right)^{-2+\alpha}(-1+\alpha) \alpha}{\sqrt{1-\left(\left(1-\mu_{A\left(x_{i}\right)}\right)^{\alpha}+\mu_{A\left(x_{i}\right)^{\alpha}}\right)^{2}}\left(\frac{\pi}{2}-\operatorname{ArcSin}\left[2^{1-\alpha}\right]\right)}
\end{aligned}
$$

Therefore, $\left.\frac{\partial^{2} H_{\alpha}^{\operatorname{Arcsin}}\left(\mu_{A}\right)}{\partial \mu_{A\left(x_{i}\right)^{2}}}\right|_{\mu_{A\left(x_{i}\right)}=0.5}=-\frac{2^{3-\alpha}(-1+\alpha) \alpha}{\sqrt{1-2^{2-2 \alpha}}\left(\frac{\pi}{2}-\operatorname{ArcSin}\left[2^{1-\alpha}\right]\right)}$, where

$$
\left.\frac{\partial^{2} H_{\alpha}^{\operatorname{Arcsin}}\left(\mu_{A}\right)}{\partial \mu_{A\left(x_{i}\right)^{2}}}\right|_{\mu_{A\left(x_{i}\right)}=0.5}<0, \forall \alpha>0 \text {. }
$$

Thus, we can see the maximum value of the fuzzy entropy (3) is attended in $\mu_{A\left(x_{i}\right)}=0.5$. Figure 5 indicates that the derivative of $H_{\alpha}^{\operatorname{Arcsin}}\left(\mu_{A}\right)$ with respect to $\mu_{A\left(x_{i}\right)}$ at value $\mu_{\mathrm{A}\left(\mathrm{x}_{\mathrm{i}}\right)}=$ 0.5 is negative for different values of $\alpha$. The maximum value of $H_{\alpha}^{C S}\left(\mu_{A}\right)$ at $\mu_{A\left(x_{i}\right)}=0.5$ is equal to 1 .
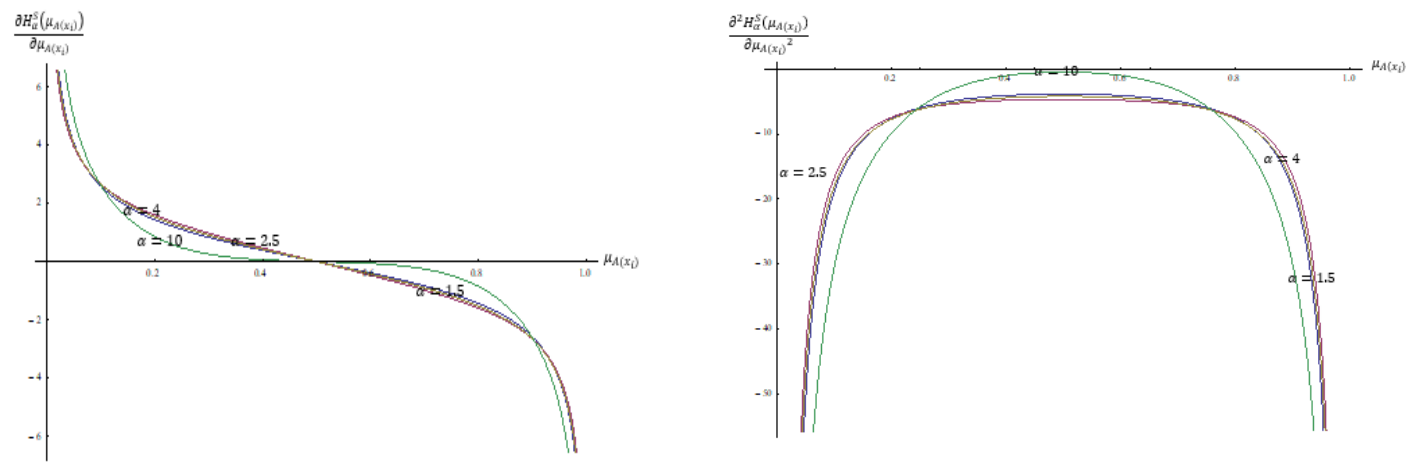

Figure 5: Plots of the first (left) and second (right) derivative of $H_{\alpha}^{\operatorname{Arcsin}}\left(\mu_{A}\right)$ for $\alpha=0.2$,

$$
0.5,1,4 \text { and } 10 .
$$

From Table 3 we found that the value of entropy $H_{\alpha=1.5}^{\operatorname{Arcsin}}\left(\mu_{A}\right)$ is increasing as $\alpha$ increasing for 2.5 to 10 for the same membership value. However, the proposed entropy measure $H_{\alpha=1.5}^{\operatorname{Arcsin}}\left(\mu_{A}\right)$ as function of $\mu_{A\left(x_{i}\right)}$ increases in the interval $[0,0.5]$ and declines in the interval $[0.5,1]$ at any given $\alpha$.

Table 3. The values of $H_{\alpha=1.5}^{A r c s i n}\left(\mu_{A}\right)$ for $\alpha=1.5,2.5,4$ and 10.

\begin{tabular}{ccccc}
\hline$\mu_{A\left(x_{i}\right)}$ & $H_{\alpha=1.5}^{\operatorname{Arcsin}}\left(\mu_{A}\right)$ & $H_{\alpha=2.5}^{\operatorname{Arcsin}}\left(\mu_{A}\right)$ & $H_{\alpha=4}^{\operatorname{Arcsin}}\left(\mu_{A}\right)$ & $H_{\alpha=10}^{\operatorname{Arcsin}}\left(\mu_{A}\right)$ \\
\hline 0.1 & 0.615435 & 0.570063 & 0.591521 & 0.774224 \\
0.2 & 0.808694 & 0.776679 & 0.793532 & 0.932671 \\
0.3 & 0.920225 & 0.904258 & 0.913181 & 0.983233 \\
0.4 & 0.980706 & 0.976451 & 0.978898 & 0.997324 \\
0.5 & 1 & 1 & 1 & 1
\end{tabular}




\begin{tabular}{ccccc}
0.6 & 0.980706 & 0.976451 & 0.978898 & 0.997324 \\
0.7 & 0.920225 & 0.904258 & 0.913181 & 0.983233 \\
0.8 & 0.808694 & 0.776679 & 0.793532 & 0.932671 \\
0.9 & 0.615435 & 0.570063 & 0.581521 & 0.774224 \\
1 & 0 & 0 & 0 & 0 \\
\hline
\end{tabular}

As Figure 6 indicates, as a function of $\alpha$, the fuzzy entropy $H_{\alpha=1.5}^{\operatorname{Arcsin}}\left(\mu_{A}\right)$ has a unique minimum at $\alpha=3$ for different values of the member function $\mu_{A\left(x_{i}\right)}$.

Resolutions: The measure $H_{\alpha}^{\operatorname{Arcsin}}\left(\mu_{A}\right)$ is a continuous concave function of $\mu_{A\left(x_{i}\right)}$ with a unique maximum at $\mu_{A\left(x_{i}\right)}=0.5$. Hence, $H_{\alpha}^{\operatorname{Arcsin}}\left(\mu_{A}\right)$ is a monotone increasing function for values $0 \leq \mu_{A\left(x_{i}\right)} \leq 0.5$ and monotone decreasing function for values $0.5 \leq \mu_{A\left(x_{i}\right)} \leq$ 1. Therefore, $H_{\alpha}^{\operatorname{Arcsin}}\left(\mu_{A}^{*}\right) \leq H_{\alpha}^{\operatorname{Arcsin}}\left(\mu_{A}\right)$ where $\mu_{A\left(x_{i}\right)}^{*}$ denotes the sharpened version of $\mu_{A\left(x_{i}\right)}$. Figure 6 presents the graph of $H_{\alpha}^{\operatorname{Arcsin}}\left(\mu_{A}\right)$ for different values: 1.5, 2.5, 4 and 10 of $\alpha$.
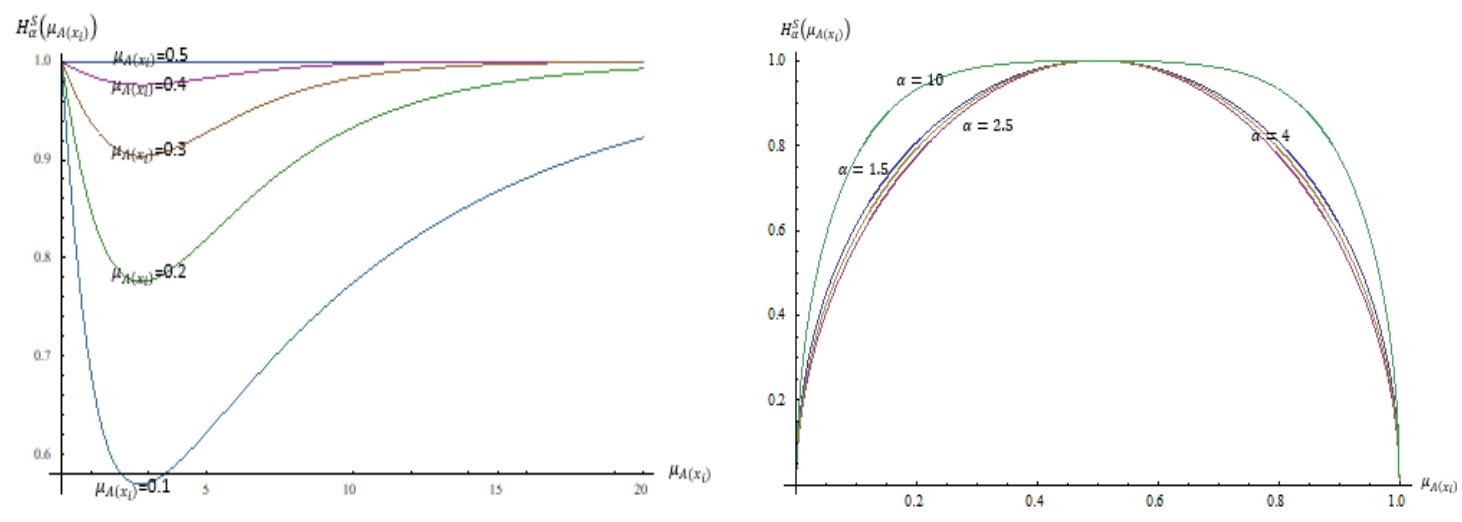

Figure 6: Plots of $H_{\alpha}^{\operatorname{Arcsin}}\left(\mu_{A}\right)$ as a function of $\alpha$ for different values of $\mu_{A\left(x_{i}\right)}$ (left) and as a function of $\mu_{A\left(x_{i}\right)}$ for $\alpha=0.2,0.5,1,4$ and 10 (right).

Symmetry: If we substitute $1-\mu_{A\left(x_{i}\right)}$ instead of $\mu_{A\left(x_{i}\right)}$ in $\operatorname{FEM}(3)$, we have

$$
H_{\alpha}^{\operatorname{Arcsin}}\left(1-\mu_{A}\right)=H_{\alpha}^{\operatorname{Arcsin}}\left(\mu_{A}\right) \text {. }
$$

Therefore, the proof of theorem 3 is achieved.

\section{A comparative measure of FEMs}

In this section, the performance of the proposed fuzzy entropy measures is studied by a numerical comparison between the proposed fuzzy entropy measures and several defined measures which are defined by authors. Firstly, this comparison is done through the trigonometry fuzzy entropy. In the second step, the comparison is done with other fuzzy entropies which are defined by De Luca and Termini (1972), Bhandari and Pal (1993), Kapur (1997) and Al-Talib and Al-Naseer (2018). The results of the comparisons are given in Tables 4 and 5, respectively.

Table 4 indicates that $H_{\alpha}^{A r c s i n}\left(\mu_{A}\right)$ produces the greatest entropy measure through other trigonometry fuzzy entropies for $\alpha$ values 1.5, 2.5, 4 and 10. Therefore, $H_{\alpha}^{\operatorname{Arcsin}}\left(\mu_{A}\right)$ is most informative than that other trigonometry fuzzy entropies for different $\alpha$ values. The results in Table 4 also indicate the following relationship 


$$
H_{\alpha}^{\operatorname{Arcsin}}\left(\mu_{A}\right)>H_{\alpha}^{\operatorname{Arctan}}\left(\mu_{A}\right)>H_{\alpha}^{S}\left(\mu_{A}\right)>H_{\alpha}^{C S}\left(\mu_{A}\right)
$$

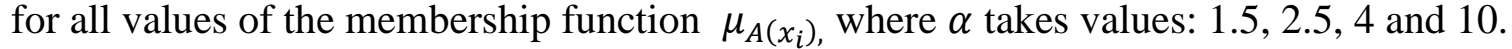
It is obvious from Table 5, the values of FEM $H_{\alpha}^{\operatorname{Arcsin}}\left(\mu_{A}\right)$ are greater than the fuzzy entropy measures of other fuzzy entropies for $\alpha=1.5,2.5,4$ and 10. Thus, $H_{\alpha}^{\operatorname{Arcsin}}\left(\mu_{A}\right)$ provides the most informative fuzzy entropy compare with other fuzzy entropies that are presented in Table 5 for values of $\alpha$ equal to 1.5, 2.5, 4 and 10. Table 5 indicates the following relationships

$$
\begin{aligned}
& H_{\alpha}^{\operatorname{Arcsin}}\left(\mu_{A}\right)>H^{D T}\left(\mu_{A}\right)>H_{\alpha}^{N T}\left(\mu_{A}\right), \\
& H_{\alpha}^{\operatorname{Arcsin}}\left(\mu_{A}\right)>H_{\alpha}^{B P}\left(\mu_{A}\right) \text { and } \\
& H_{\alpha}^{\operatorname{Arcsin}}\left(\mu_{A}\right)>H_{\alpha}^{K}\left(\mu_{A}\right)
\end{aligned}
$$

are satisfied for values $\alpha=1.5,2.5,4,10$ when different values of the membership

\begin{tabular}{|c|c|c|c|c|}
\hline$\mu_{A\left(x_{i}\right)}$ & $H_{\alpha=1.5}^{S}\left(\mu_{A}\right)$ & $H_{\alpha=1.5}^{C S}\left(\mu_{A}\right)$ & $H_{\alpha=1.5}^{\operatorname{Arctan}}\left(\mu_{A}\right)$ & $H_{\alpha=1.5}^{\operatorname{Arcsin}}\left(\mu_{A}\right)$ \\
\hline 0 & 0 & 0 & 0 & 0 \\
\hline 0.1 & 0.229707 & 0.17178 & 0.357154 & 0.615435 \\
\hline 0.2 & 0.524668 & 0.450638 & 0.633394 & 0.808694 \\
\hline 0.3 & 0.776622 & 0.727673 & 0.835169 & 0.920255 \\
\hline 0.4 & 0.942415 & 0.927491 & 0.958489 & 0.980706 \\
\hline 0.5 & 1 & 1 & 1 & 1 \\
\hline$\mu_{A\left(x_{i}\right)}$ & $H_{\alpha=2.5}^{S}\left(\mu_{A}\right)$ & $H_{\alpha=2.5}^{C S}\left(\mu_{A}\right)$ & $H_{\alpha=2.5}^{\operatorname{Arctan}}\left(\mu_{A}\right)$ & $H_{\alpha=2.5}^{\operatorname{Arcsin}}\left(\mu_{A}\right)$ \\
\hline 0 & 0 & 0 & 0 & 0 \\
\hline 0.1 & 0.130836 & 0.0530831 & 0.287768 & 0.570063 \\
\hline 0.2 & 0.407414 & 0.264879 & 0.565860 & 0.776679 \\
\hline 0.3 & 0.702354 & 0.588700 & 0.796460 & 0.904528 \\
\hline 0.4 & 0.92029 & 0.882096 & 0.94759 & 0.976451 \\
\hline 0.5 & 1 & 1 & 1 & 1 \\
\hline$\mu_{A\left(x_{i}\right)}$ & $H_{\alpha=4}^{S}\left(\mu_{A}\right)$ & $H_{\alpha=4}^{C S}\left(\mu_{A}\right)$ & $H_{\alpha=4}^{\operatorname{Arctan}}\left(\mu_{A}\right)$ & $H_{\alpha=4}^{\operatorname{Arcsin}}\left(\mu_{A}\right)$ \\
\hline 0 & 0 & 0 & 0 & 0 \\
\hline 0.1 & 0.0954915 & 0.00911863 & 0.30962 & 0.591521 \\
\hline 0.2 & 0.345492 & 0.119364 & 0.597955 & 0.793532 \\
\hline 0.3 & 0.654508 & 0.428381 & 0.820089 & 0.913181 \\
\hline 0.4 & 0.904508 & 0.818136 & 0.955197 & 0.978898 \\
\hline 0.5 & 1 & 1 & 1 & 1 \\
\hline$\mu_{A\left(x_{i}\right)}$ & $H_{\alpha=10}^{S}\left(\mu_{A}\right)$ & $H_{\alpha=10}^{C S}\left(\mu_{A}\right)$ & $H_{\alpha=10}^{\operatorname{Arctan}}\left(\mu_{A}\right)$ & $H_{\alpha=10}^{\operatorname{Arcsin}}\left(\mu_{A}\right)$ \\
\hline 0 & 0 & 0 & 0 & 0 \\
\hline 0.1 & 0.124282 & $7.94006 \times 10^{-6}$ & 0.57426 & 0.774224 \\
\hline 0.2 & 0.420867 & 0.00492251 & 0.865962 & 0.932671 \\
\hline 0.3 & 0.729884 & 0.120109 & 0.96644 & 0.983233 \\
\hline 0.4 & 0.933299 & 0.605429 & 0.994641 & 0.997324 \\
\hline 0.5 & 1 & 1 & 1 & 1 \\
\hline
\end{tabular}
function $\mu_{A\left(x_{i}\right)}$ are applied.

Table 4: Comparison of the trigonometry fuzzy entropies (maximum values are bolded). 


\section{Conclusions}

In this paper, three new trigonometric fuzzy entropies of order $\alpha$ are proposed. We found that the four axiomatic requirements properties are satisfied with the new fuzzy entropies. The results of the preceding sections confirm that the FEM $H_{\alpha}^{A r c s i n}, \alpha>1$ produces the greatest entropy value not only through some proposed $\alpha$-order trigonometric fuzzy entropies but also, it produces the highest entropy value through some non-trigonometric fuzzy entropies which were suggested in previous studies. Another possible topic for future research is to use entropy of order $\alpha$ in the fuzzy setting for multi criteria decision making problems (Adel Rastkhiz, 2019) which has an application in evaluating entrepreneurial opportunities.

Table 5: Comparison of the $H_{\alpha}^{A r c s i n}\left(\mu_{A}\right)$ fuzzy entropy with non- trigonometry fuzzy entropies (maximum values are bolded).

\begin{tabular}{|c|c|c|c|c|c|}
\hline$\mu_{A\left(x_{i}\right)}$ & $H_{\alpha=1.5}^{\operatorname{Arcsin}}\left(\mu_{A}\right)$ & $H^{D P}\left(\mu_{A}\right)$ & $H_{\alpha=1.5}^{N T}\left(\mu_{A}\right)$ & $H_{\alpha=1.5}^{B P}\left(\mu_{A}\right)$ & $H_{\alpha=1.5}^{K}\left(\mu_{A}\right)$ \\
\hline 0 & 0 & 0 & 0 & 0 & 0 \\
\hline 0.1 & 0.615435 & 0.468996 & 0.419271 & 0.351074 & 0.39114 \\
\hline 0.2 & 0.808694 & 0.721928 & 0.638987 & 0.625934 & 0.665825 \\
\hline 0.3 & 0.920255 & 0.881291 & 0.821277 & 0.830157 & 0.853626 \\
\hline 0.4 & 0.980706 & 0.970951 & 0.951512 & 0.956933 & 0.963695 \\
\hline 0.5 & 1 & 1 & 1 & 1 & 1 \\
\hline$\mu_{A\left(x_{i}\right)}$ & $H_{\alpha=2.5}^{\operatorname{Arcsin}}\left(\mu_{A}\right)$ & $H^{D T}\left(\mu_{A}\right)$ & $H_{\alpha=2.5}^{N T}\left(\mu_{A}\right)$ & $H_{\alpha=2.5}^{B P}\left(\mu_{A}\right)$ & $H_{\alpha=2.5}^{K}\left(\mu_{A}\right)$ \\
\hline 0 & 0 & - & 0 & 0 & 0 \\
\hline 0.1 & 0.570063 & 0.468996 & 0.417007 & 0.249389 & 0.353323 \\
\hline 0.2 & 0.776679 & 0.721928 & 0.634068 & 0.506951 & 0.633738 \\
\hline 0.3 & 0.904528 & 0.881291 & 0.816223 & 0.748415 & 0.836483 \\
\hline 0.4 & 0.976451 & 0.970951 & 0.949413 & 0.930498 & 0.959015 \\
\hline 0.5 & 1 & 1 & 1 & 1 & 1 \\
\hline$\mu_{A\left(x_{i}\right)}$ & $H_{\alpha=4}^{\operatorname{Arcsin}}\left(\mu_{A}\right)$ & $H^{D T}\left(\mu_{A}\right)$ & $H_{\alpha=4}^{N T}\left(\mu_{A}\right)$ & $H_{\alpha=4}^{B P}\left(\mu_{A}\right)$ & $H_{\alpha=4}^{K}\left(\mu_{A}\right)$ \\
\hline 0 & 0 & - & 0 & 0 & 0 \\
\hline 0.1 & 0.591521 & 0.468996 & 0.41246 & 0.202598 & 0.202598 \\
\hline 0.2 & 0.793532 & 0.721928 & 0.623152 & 0.427363 & 0.427363 \\
\hline 0.3 & 0.913181 & 0.881291 & 0.803519 & 0.670142 & 0.671042 \\
\hline 0.4 & 0.978898 & 0.970951 & 0.943452 & 0.895933 & 0.895933 \\
\hline 0.5 & 1 & 1 & 1 & 1 & 1 \\
\hline$\mu_{A\left(x_{i}\right)}$ & $H_{\alpha=10}^{\operatorname{Arcsin}}\left(\mu_{A}\right)$ & $H^{D T}\left(\mu_{A}\right)$ & $H_{\alpha=10}^{N T}\left(\mu_{A}\right)$ & $H_{\alpha=10}^{B P}\left(\mu_{A}\right)$ & $H_{\alpha=10}^{K}\left(\mu_{A}\right)$ \\
\hline 0 & 0 & - & 0 & 0 & 0 \\
\hline 0.1 & 0.774224 & 0.468996 & 0.40645 & 0.168892 & 0.652596 \\
\hline 0.2 & 0.932671 & 0.721928 & 0.60559 & 0.357698 & 0.894373 \\
\hline 0.3 & 0.983233 & 0.881291 & 0.777019 & 0.571714 & 0.973648 \\
\hline 0.4 & 0.997324 & 0.970951 & 0.924995 & 0.816095 & 0.995793 \\
\hline 0.5 & 1 & 1 & 1 & 1 & 1 \\
\hline
\end{tabular}

\section{Acknowledgment}

The authors would like to thanks the Editor and the respectful referees for their contributional comments to improve the article significantly. 


\section{References}

1. Adel Rastkhiz, S. E., Mobini Dehkordi, A., Yadollahi Farsi, J. and Azar, A. (2019). A new approach to evaluating entrepreneurial opportunities. Journal of Small Business and Enterprise Development, 26(1), 67-84.

2. Al-Talib, M. and Al-Nasser, A. (2018). New Fuzzy Entropy Measure of Order $\alpha$. Pakistan Journal of Statistics and Operation Research, 14(4), 831-838.

3. Bhandari, D. and Pal, N. R. (1993). Some new information measures for fuzzy sets. Information Sciences, 67(3), 209-228.

4. Campión, M. J., Catalán, R. G., Induráin, E. and Valero, O. (2018). Weightable quasi-metrics related to fuzzy sets. Hacettepe Journal of Mathematics and Statistics, 47(5), 1184-1195.

5. Chatterjee, A., Singh, G. N., Bandyopadhyay, A. and Mukhopadhyay, P. (2017). A general procedure for estimating population variance in successive sampling using fuzzy tools. Hacettepe Journal of Mathematics and Statistics, 46(4), 695-712.

6. De Luca, A. and Termini, S. (1972). A definition of a nonprobabilistic entropy in the setting of fuzzy sets theory. Information and control, 20(4), 301-312.

7. Das, S., Guha, D. and Dutta, B. (2016). Medical diagnosis with the aid of using fuzzy logic and intuitionistic fuzzy logic. Applied Intelligence, 45(3), 850-867.

8. Fan, J. L. and Ma Y. L. (2002). Some new fuzzy entropy formulas. Fuzzy sets and Systems, 128(2), 277-284.

9. Gupta, P. and Sheoran, A. (2014). Harmonic Measures of Fuzzy Entropy and their Normalization. International Journal of Software and Applied Mathematics, 10 (3), 4751, 2014.

10. Hooda, D. S. and Mishra, A. R. (2015). On trigonometric fuzzy information measures. ARPN Journal of Science and Technology, 5(3), 145-152.

11. $\mathrm{Hu}, \mathrm{Q}$. and $\mathrm{Yu}, \mathrm{D}$. (2004). Entropies of fuzzy indiscernibility relation and its operations. International Journal of uncertainty, fuzziness and knowledge-based systems, 12(05), 575-589.

12. Kapur, J. N. (1997). Measures of fuzzy information. Mathematical Sciences Trust Society.

13. Parkash, O., Sharma, P. K. and Mahajan, R. (2008). New measures of weighted fuzzy entropy and their applications for the study of maximum weighted fuzzy entropy principle. Information Sciences, 178(11), 2389-2395.

14. Rényi, A. (1961). On measures of entropy and information. In Proceedings of the Fourth Berkeley Symposium on Mathematical Statistics and Probability, Volume 1: Contributions to the Theory of Statistics. The Regents of the University of California.

15. Shannon, C. E. (1948). A Mathematical Theory of Communication, Bell System Technical Journal, 27, 379-423.

16. Verma, R. (2015). Cosine entropy and similarity measures for fuzzy sets. Konuralp Journal of Mathematics, 3(1), 83-93. 
17. Zamanzade, E., Arghami, N. R. (2011). Goodness-of-fit test based on correcting moments of modified entropy estimator. Journal of Statistical Computation and Simulation, 81(12), 2077-2093.

18. Zamanzade, E., Arghami, N. R. (2012). Testing normality based on new entropy estimators. Journal of Statistical Computation and Simulation, 82(11), 1701-1713.

19. Zamanzade, E. (2014). Testing uniformity based on new entropy estimators. Journal of Statistical Computation and Simulation, 85(16), 3191-3205.

20. Zamanzade, E., Mahdizadeh, M. (2016). Entropy estimation from judgement post stratified data. Pakistan Journal of Statistics and Operation Research, 7(4), 625637.

21. Zamanzade, E., Mahdizadeh, M. (2017). Entropy estimation from ranked set samples with application to test of fit. Revista Colombiana de Estadstica, 40(2), 223-241.

22. Zadeh, L. A. (1965). Fuzzy sets, Information and Control, 8, 338-353.

23. Zadeh, L. A. (1968). Probability measures of fuzzy events. The Journal of Mathematical Analysis and Applications, 23(2), 421-427. 